\title{
Recent progress of a code system DEURACS toward deuteron nuclear data evaluation
}

\author{
Shinsuke Nakayama ${ }^{1, *}$, Osamu Iwamoto $^{1}$, and Yukinobu Watanabe ${ }^{2}$ \\ ${ }^{1}$ Nuclear Data Center, Japan Atomic Energy Agency, Ibaraki 319-1195, Japan \\ ${ }^{2}$ Department of Advanced Energy Engineering Science, Kyushu University, Fukuoka 816-8580, Japan
}

\begin{abstract}
Toward deuteron nuclear data evaluation, we have been developing a code system dedicated for deuteron-induced reactions, called DEURACS. In this paper, we review the recent progress in the development of DEURACS. To meet increasing and diversifying demands for deuteron nuclear data, DEURACS has been developed aiming at accurate and comprehensive prediction of deuteron-induced reactions. We present the results of some comparisons between the DEURACS calculations and experimental data for a variety of deuteron-induced reactions at incident energies up to $200 \mathrm{MeV}$. Double-differential cross sections for emission of neutrons or light ions up to $A=4$ and cross sections for production of residual nuclei are analyzed. Neutron yields from deuteron bombardment on thick targets are also analyzed by the Monte Carlo transport simulation based on the deuteron nuclear data provided with DEURACS. Through comparison with experimental and other calculated data, validation of the present modeling in DEURACS is discussed.
\end{abstract}

\section{Introduction}

Intensive neutron sources using $(d, x n)$ reactions on light elements (e.g. $\mathrm{Li}, \mathrm{Be}$, and $\mathrm{C}$ ) have been proposed for various applications such as irradiation testing for fusion reactor materials [1] and radioisotope production for medical and other purposes [2,3]. Moreover, transmutation system using deuteron-induced spallation reactions has been recently proposed for several long-lived fission products (LLFPs) $[4,5]$. In relations to these applications, there are various needs for deuteron nuclear data. Accurate data for double-differential cross sections (DDXs) for $(d, x n)$ reactions on light nuclei are necessary for the design of neutron sources. Cross-section data for the production of residual nuclei are also important to evaluate transmutation efficiency of LLFPs and to estimate induced radioactivity in accelerator structural materials. Data for composite particle emission are also needed to estimate production of tritium and helium gases in structural materials. However, currently available experimental and evaluated data of deuteron-induced reactions are not necessarily enough for the requirement. Hence, theoretical predictions play a key role in completing the required cross-section data by interpolation and extrapolation of experimental data. Under the above situations, we have been developing a code system for deuteron-induced reactions, called DEURACS.

Until the last conference, ND2016, we have focused on the description of nucleon emission so as to contribute to the design of neutron sources. As a result, DEURACS was successfully applied to the $(d, x p)$ and $(d, x n)$ reactions up to $100 \mathrm{MeV}$ [6-8]. In this paper, we review the recent progress in the development of DEURACS after ND2016.

*e-mail: nakayama.shinsuke@jaea.go.jp
The main progresses are as follows: (1) extension of the incident energy range to $200 \mathrm{MeV}$ in the analysis of $(d, x n)$ reactions, (2) improvement in prediction accuracy of composite particle emission, (3) application to the production of residual nuclei, and (4) development and validation of the deuteron nuclear data based on the DEURACS calculation. In the remainder of this paper, we describe each of the above items after the brief overview of DEURACS.

\section{Overview of DEURACS}

The deuteron is a weakly bound nucleus and easily breaks up through interaction with a target nucleus. Therefore, DEURACS consists of several calculation codes based on theoretical models to describe respective reaction mechanisms that are characteristic of deuteron-induced reactions. The breakup processes, namely elastic breakup and non-elastic breakup are calculated using the codes based on the continuum-discretized coupled-channels (CDCC) theory [9] and the Glauber model with the quantum $S$ matrices given by the optical model calculations [10], respectively. The $(d, n)$ and $(d, p)$ transfer reactions to the specific bound states in the residual nucleus are calculated by a conventional zero-range distorted wave Born approximation (DWBA) using the DWUCK4 code [11]. The semiempirical model by Kalbach [12] with the some modifications has recently been incorporated to describe pickup reaction in direct process [13].

In addition to the above-mentioned direct process, the pre-equilibrium and the compound nucleus processes are calculated using the two-component exciton model and the Hauser-Feshbach model implemented in the CCONE 
code $[14,15]$. For better prediction of composite particle emission, the Iwamoto-Harada model $[16,17]$ of cluster emission is incorporated with the exciton model. In deuteron-induced reactions, three types of composite nuclei can be formed by the absorption of either neutron or proton in the incident deuteron, or the deuteron itself. In DEURACS, a calculation taking these effects into account is performed. More details about the models and methods in DEURACS are described in Refs. [6, 7, 13, 18]. The choice and adjustment of model parameters are also described in the references.

\section{Results and discussion}

\subsection{Double-differential cross section for particle emission}

Recently, a new measurement of DDXs of $(d, x n)$ reactions has been performed at an incident energy of $200 \mathrm{MeV}$ [19] higher than around $100 \mathrm{MeV}$ [20]. Therefore, we analyze the DDXs of the reaction to investigate the applicability of DEURACS at the higher incident energy. Figure 1 presents the calculated and experimental DDXs of the $(d, x n)$ reaction on natural lithium $\left(92.5 \%{ }^{7} \mathrm{Li}\right.$ and $7.5 \%$ $\left.{ }^{6} \mathrm{Li}\right)$ at $200 \mathrm{MeV}$. The emission angle is $0^{\circ}$. The DEURACS calculation is shown by the solid curves. The target is assumed to be ${ }^{7} \mathrm{Li}$ in the DEURACS calculation.

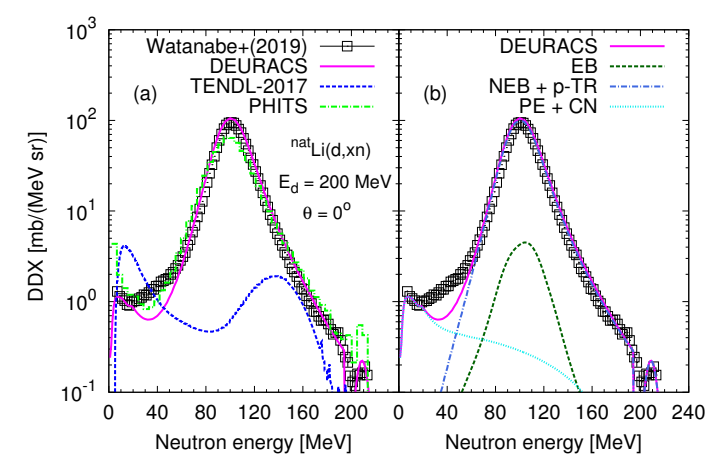

Figure 1. Calculated and experimental DDXs for the $(d, x n)$ reaction on natural lithium at $200 \mathrm{MeV}$. Comparison of the DEURACS calculation with the experimental and other calculated data is presented in (a), and the components corresponding to each reaction process in the DEURACS calculation are shown in (b). Open squares are the experimental data taken from Ref. [19]. Note that only in the DEURACS calculation the target is assumed to be ${ }^{7} \mathrm{Li}$. See text for the meaning of the curves.

In Fig. 1(a), we also show the values stored in the deuteron sub-library of TENDL-2017 [21] and the calculated values with the PHITS code [22] by the dashed and the dash-dotted lines, respectively. TENDL is the nuclear data library based on the calculation of the TALYS code. The models employed in the present PHITS calculation are INCL-4.6 [23] for the dynamical processes including breakup reactions, and GEM [24] for the evaporation process that subsequently occurs. As shown in the figure, DEURACS reproduces the experimental data better than the other results (TENDL-2017 and PHITS) especially at the broad peak around half the deuteron incident energy. Together with the results of the previous analyses up to around $100 \mathrm{MeV}[7,8]$, these results demonstrate the applicability of DEURACS to $(d, x n)$ reactions on light nuclei at incident energies up to $200 \mathrm{MeV}$. However, it should be mentioned that the applicability of the Glauber model gets worse for high-atomic-number nuclei such as ${ }^{208} \mathrm{~Pb}$ and ${ }^{238} \mathrm{U}$ as mentioned in Ref. [10].

To understand the result of Fig. 1(a) more deeply, we show in Fig. 1(b) the components corresponding to each reaction process in the DEURACS calculation. The dashed, the dash-dotted, and the dotted curves represent the components of elastic breakup (EB), nonelastic breakup (NEB) and proton transfer ( $p$-TR), and preequilibrium $(\mathrm{PE})$ and compound nucleus $(\mathrm{CN})$ processes, respectively. The characteristic peak around half the deuteron incident energy is formed mainly by the nonelastic breakup. Note that the proton transfer $(d, n)$ reactions contribute only to the sharp peak in the high emission energy region. This suggests that the model in DEURACS for the nonelastic breakup is more adequate than those in the other calculations, namely Kalbach's empirical model [25] in TALYS and the INCL model in PHITS.

Nest, we analyze the composite particle emission. Figures 2 and 3 show the results for DDXs of the $(d, x t)$ and $(d, x \alpha)$ reactions on ${ }^{27} \mathrm{Al}$ at $80 \mathrm{MeV}$, respectively. Same as Fig. 1, the values of TENDL-2017 and PHITS are also presented in Figs. 2(a) and 3(a), and the components corresponding to each reaction process in the DEURACS calculation are shown in Figs. 2(b) and 3(b). The dashed and the dotted curves represent the components of direct pickup (PU), and pre-equilibrium (PE) and compound nucleus $(\mathrm{CN})$ processes in DEURACS, respectively.

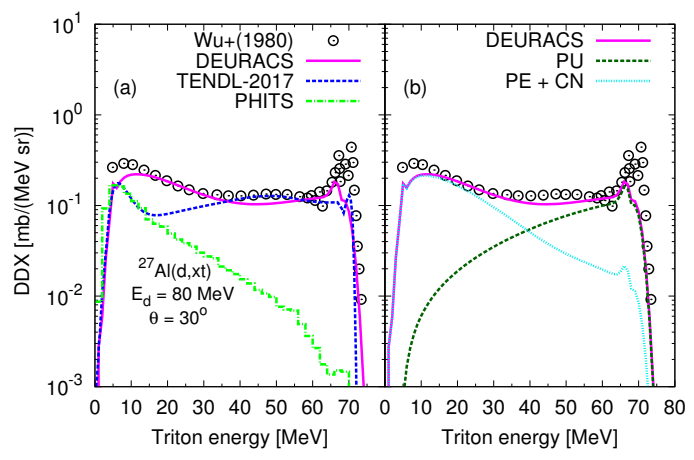

Figure 2. Same as figure 1 but for the ${ }^{27} \mathrm{Al}(d, x t)$ reaction at 80 $\mathrm{MeV}$. Experimental data are taken from Ref. [26].

In contrast to TENDL-2017 and PHITS, DEURACS reproduces the experimental data in the wide emission energy range, as illustrated in Figs. 2(a) and 3(a). These results are achieved by the inclusion of the model by Iwamoto and Harada describing pre-equilibrium cluster emission and the semi-empirical model by Kalbach describing direct pickup process. On the other hand, some discrepancies between DEURACS and the experimental data are seen in the highest energy region. This is due to that the components of the direct pickup reactions for specific bound states in the residual nucleus are absent in the 
present DEURACS calculation. The problem is caused by lack of experimental data necessary for extracting spectroscopic factors, which determine the absolute values of these components. Enrichment of experimental data for the angular distributions of the $(d, t)$ and $(d, \alpha)$ reactions to the low-lying states in the residual nucleus is expected.

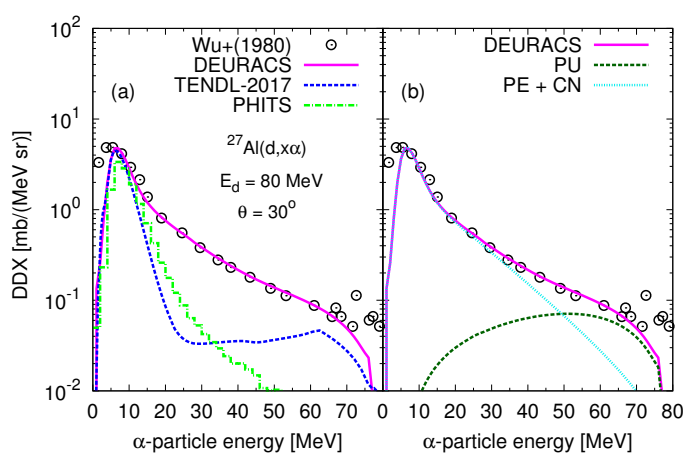

Figure 3. Same as figure 1 but for the ${ }^{27} \mathrm{Al}(d, x \alpha)$ reaction at 80 $\mathrm{MeV}$. Experimental data are taken from Ref. [26].

\subsection{Cross section for production of residual nuclei}

Figure 4 illustrates the calculated and experimental cross sections of the ${ }^{27} \mathrm{Al}(d, x)^{22} \mathrm{Na}$ reaction at the incident energies up to $100 \mathrm{MeV}$. As shown in Fig. 4(a), the DEURACS calculation is in a good agreement with the experimental data in the whole incident energy range. TENDL-2017 reproduces the experimental data only in the low energy range below $45 \mathrm{MeV}$, and the PHITS calculation underestimates the experimental data in the whole range.

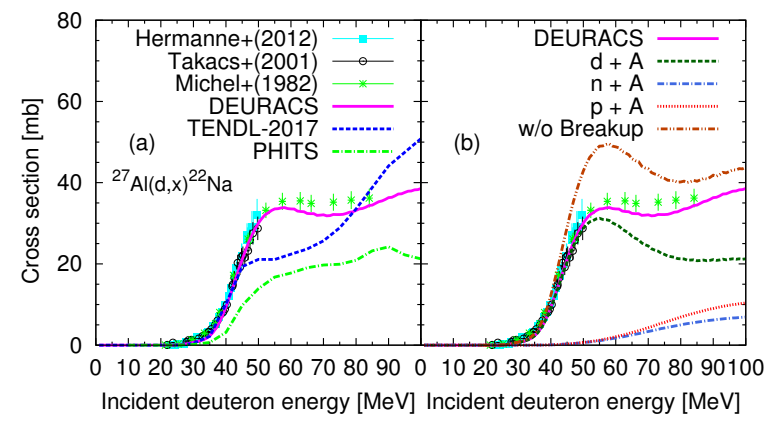

Figure 4. Calculated and experimental cross sections for the ${ }^{27} \mathrm{Al}(d, x)^{22} \mathrm{Na}$ reaction. Comparison of the DEURACS calculation with the experimental and other calculated data is presented in (a). The contributions from the absorption of deuteron, proton, and neutron in DEURACS are shown in (b). The calculated result with DEURACS in which the breakup processes are not considered is also shown in (b). Experimental data are taken from Refs. [27-29]. See text for the meaning of the curves.

To assess the effect of breakup processes in the production of residual nuclei, we decompose the calculated values of DEURACS into the three components, namely the contributions from three different composite nuclei formed by the absorption of either neutron or proton in the incident deuteron, or the deuteron itself. They are shown in Fig. 4(b). The dashed, the dash-dotted, and the dotted curves in the figure represent the contributions from the absorption of deuteron, proton, and neutron, respectively. We also show the result of the DEURACS calculation in which the breakup processes are not considered by the dash-dot-dotted line. The calculation without breakup overestimates the experimental data. On the other hand, by considering the breakup processes the deuteron absorption cross section is decreased and the component of deuteron absorption $(d+A)$ gets smaller than the values calculated without breakup. In addition, the components from the absorption of neutron $(n+A)$ and proton $(p+A)$ appear, and consequently the sum of the three components reproduces the experimental data well. This result demonstrates that consideration of the breakup processes is important also in the calculation for the production of residual nuclei.

Figure 5 shows the isotopic distribution of rhodium produced from the ${ }^{107} \mathrm{Pd}+d$ reaction at $200 \mathrm{MeV}$. Since the measurement using the inverse kinematic method has been performed in Ref. [4], the comprehensive data including the one for the stable ${ }^{103} \mathrm{Rh}$ are available. As shown in Fig. 5(a), DEURACS reproduce the experimental data in the wide mass number range. In addition, the influence of the consideration of the breakup processes is also seen in Fig. 5(b). In contrast to Fig. 4(a), the PHITS calculation is in good agreement with the experimental data. This may be due to that the assumption in an intra-nuclear cascade approach gets better in the high incident energy of $200 \mathrm{MeV}$, i.e., $100 \mathrm{MeV} /$ nucleon. TENDL-2017 show serious disagreement with the experimental data especially in the smaller mass number region. Together with the result of Fig. 4, it can be said that only DEURACS can give a reliable prediction at the incident energies up to $200 \mathrm{MeV}$.

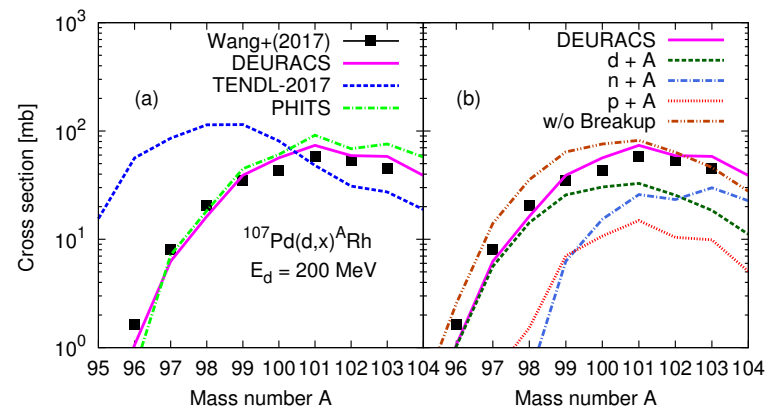

Figure 5. Same as figure 4 but for the rhodium isotopes production from the ${ }^{107} \mathrm{Pd}(d, x)$ reaction at $200 \mathrm{MeV}$. Experimental data are taken from Ref. [4].

\subsection{Neutron yield from thick target}

To make use of the result of DEURACS in the Monte Carlo transport simulation, we have developed the deuteron nuclear data based on the DEURACS calculation. In the development of the nuclear data, we have adopted the "Frag Data" format uniquely defined in the PHITS code. The target nuclei are ${ }^{7} \mathrm{Li},{ }^{9} \mathrm{Be}$, and ${ }^{12} \mathrm{C}$, and the incident energy range is from 1 to $200 \mathrm{MeV}$. We have compiled the DDXs 
for the emission of each particle $\left(n, p, d, t,{ }^{3} \mathrm{He}\right.$, and $\left.\alpha\right)$ and $\gamma$-ray in the format of "Frag Data".

To investigate the validity of the transport simulation using the deuteron nuclear data, we analyze the neutron yields from deuteron bombardment on thick targets. Figure 6 illustrates the calculated and experimental thick-target neutron yields (TTNYs) from deuteron bombardment on (a) natural lithium at $40 \mathrm{MeV}$ and (b) ${ }^{12} \mathrm{C}$ at $18 \mathrm{MeV}$. Following the experimental conditions in Refs. [30, 31], the target thicknesses are set to be $21.4 \mathrm{~mm}$ for lithium and $1.0 \mathrm{~mm}$ for carbon, respectively. The solid lines are the calculation results using the deuteron nuclear data. Note that only the cross sections of the deuteroninduced reactions on ${ }^{7} \mathrm{Li}$ and ${ }^{12} \mathrm{C}$ follow the data, and other reactions are described by the models and data implemented in PHITS. The dash-dotted lines are the simulation results using the PHITS code alone. In the present PHITS calculation, the approach combining INCL and DWBA developed by Hashimoto et al. [32] is adopted for $(d, x n)$ reactions. As shown in the figure, the simulation results based on the nuclear data reproduce the experimental data better than the results of PHITS. These results demonstrate the validity of the nuclear data developed with DEURACS.

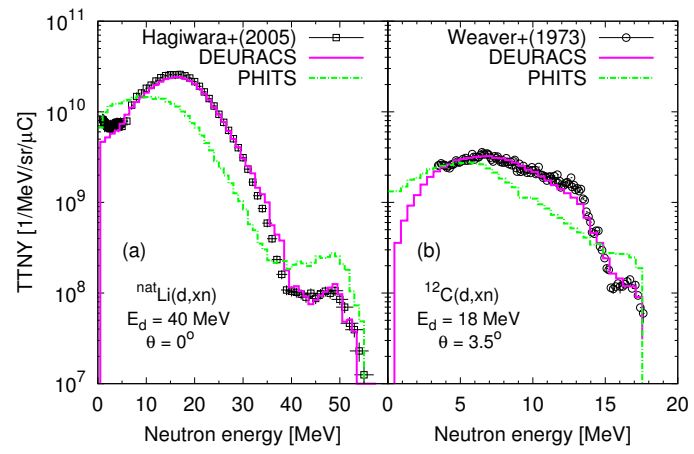

Figure 6. Calculated and experimental TTNYs from deuteron bombardment on (a) natural lithium at $40 \mathrm{MeV}$ and (b) ${ }^{12} \mathrm{C}$ at 18 $\mathrm{MeV}$. Experimental data taken from Refs. [30, 31], respectively. See text for the meaning of the curves.

\section{Summary and outlook}

We have presented some resent progresses in the development of DEURACS. The DEURACS calculations have reproduced experimental data for a variety of deuteroninduced reactions at incident energies up to $200 \mathrm{MeV}$. Based on the DEURACS results, we have developed the deuteron nuclear data on light nuclei dedicated for the PHITS code. The nuclear data has been validated from the analysis for thick-target neutron yields using the Monte Carlo transport simulation.

In the future, we plan to make ENDF-6 formatted nuclear data so that the DEURACS results can be used in Monte Carlo codes other than PHITS, such as MCNP.

\section{Acknowledgment}

This work was partially supported by the ImPACT Program of Council for Science, Technology and Innovation (Cabinet Office, Government of Japan).

\section{References}

[1] A. Moeslang et al., Fusion Eng. Des. 81, 863 (2006)

[2] Y. Nagai et al., J. Phys. Soc. Jpn. 82, 064201 (2013)

[3] T. Kin et al. (2019), presented at this conference

[4] H. Wang et al., Prog. Theor. Exp. Phys. 2017, 021D01 (2017)

[5] S. Kawase et al., Prog. Theor. Exp. Phys. 2017, 093D03 (2017)

[6] S. Nakayama, Y. Watanabe, J. Nucl. Sci. Technol. 53, 89 (2016)

[7] S. Nakayama et al., Phys. Rev C 94, 014618 (2016)

[8] S. Nakayama et al., EPJ Web Conf. 146, 12025 (2017)

[9] M. Yahiro et al., Prog. Theor. Exp. Phys. 2012, 01A206 (2012), and references therein

[10] T. Ye et al., Phys. Rev. C 84, 054606 (2011)

[11] P.D. Kunz, Computer code DWUCK4, University of Colorado (1974), unpublished

[12] C. Kalbach, Z. Phys. A 283, 401 (1977)

[13] S. Nakayama et al., Phys. Rev C 100, 044603 (2019)

[14] O. Iwamoto, J. Nucl. Sci. Technol. 44, 687 (2007)

[15] O. Iwamoto et al., Nucl. Data Sheets 131, 259 (2016)

[16] A. Iwamoto et al., Phys. Rev. C 26, 1821 (1982)

[17] S. Kunieda et al., Phys. Rev. C 85, 054602 (2012)

[18] S. Nakayama et al., Phys. Rev C 98, 044606 (2018)

[19] Y. Watanabe et al. (2019), presented at this conference

[20] S. Araki et al., Nucl. Instr. Meth. A 842, 62 (2017)

[21] A.J. Koning et al., Nucl. Data Sheets 155, 1 (2019)

[22] T. Sato et al., J. Nucl. Sci. Technol. 55, 684 (2018)

[23] A. Boudard et al., Phys. Rev C 87, 014606 (2013)

[24] S. Furihata, Nucl. Instr. Meth. B 171, 251 (2000)

[25] C. Kalbach, Phys. Rev. C 95, 014606 (2017)

[26] J.R. Wu et al., Phys. Rev. C 19, 370 (1979)

[27] A. Hermanne et al., Appl. Radiat. Isot. 70, 2763 (2012)

[28] S. Takács et al., Nucl. Instr. Meth. B 174, 235 (2001)

[29] R. Michel et al., private communication (1982), EXFOR Entry No. A0158, dated 2004-03-29

[30] M. Hagiwara et al., Fusion Sci. Technol. 48, 1320 (2005)

[31] K.A. Weaver et al., Nucl. Sci. Eng. 52, 35 (1973)

[32] S. Hashimoto et al., Nucl. Instr. Meth. B 333, 27 (2014) 